\title{
Promote Students' Healthy Behavior Through Sensor and Game: A Randomized Controlled Trial
}

\author{
Florence H-Jennings $^{1} \cdot$ Marie-Véronique Clément $^{1} \cdot$ Michael Brown $^{1} \cdot$ Ben Leong $^{1}$ • \\ Liang Shen ${ }^{1} \cdot$ Chaoyan Dong ${ }^{1}$
}

Published online: 3 May 2016

(C) The Author(s) 2016. This article is published with open access at Springerlink.com

\begin{abstract}
Objectives The aim of the study was to assess the impact of using sensor technology to track steps and sleep with and without a game platform in motivating students to attain the recommended exercise level and sleep hours.

Methods Three hundred first-year medical students were randomly assigned to the control group, group 1 using a tracking device (FitBit Flex) and group 2 using FitBit Flex and a social media-based game. Stress, exercise, and sleep time and quality were assessed by validated instruments. Daily steps and sleep hours were monitored by FitBit Flex from week 1 to week 8 in group 1 and group 2. From week 9, all groups had access to FitBit Flex and the game platform, and students' daily steps and sleep time were monitored until week 14 by FitBit Flex.

Results For exercise, $85 \%$ of students were active at the baseline, and the interventions did not significantly modify their exercise level except during recess and exam week where the incentive of the game mitigated the decrease of daily steps. For sleep, by week 14, all groups attained an average of daily sleep time close to $8 \mathrm{~h}$ compared to the baseline of $7.26 \mathrm{~h}$. For stress, baseline survey showed that $57.7 \%$ of students experienced medium to high stress level, and this trend did not significantly change during the study.

Conclusion It is more important to reinforce the benefit of staying active instead of motivating students to start doing exercise. Tracking sleep is critical to motivate a student to increase sleep time close to the recommended $8 \mathrm{~h}$.
\end{abstract}

Marie-Véronique Clément

marie-veronique clement@nuhs.edu.sg

1 Yong Loo Lin School of Medicine, National University of Singapore, Singapore, Singapore
Keywords Medical students $\cdot$ Health $\cdot$ Stress $\cdot$ Sleep $\cdot$ Steps

\section{Introduction}

Although medical students are similar to the general student population before medical school [1], stress becomes increasingly prevalent after training begins [2]. According to a recent systematic review [3], half of US medical students are affected by chronic stress. Dyrbye et al. suggested exploring organizational interventions "to address the curricular, training, and system factors that also contribute to the high prevalence of distress" [4]. Coherence between curriculum and systematic support for medical students is necessary for their future wellbeing [5]. Medical education needs to prepare students for the increased demands of healthcare systems, which will affect their health and performance so as to mitigate the effects of burnout and fatigue. Chronic stress not only affects students' mental well-being but also cognitive abilities, which have been found related to physiological changes. The hippocampus is particularly exposed to the deregulation of glucocorticoid production, potentially contributing to hippocampal atrophy and long-term deficits in memory and cognition [6-8].

Physicians' chronic stress and burnout have been found to correlate with increased incidences of medical errors [9-11], medical malpractice suits $[12,13]$, diminished levels of patient compliance $[14,15]$, reduced patient satisfaction [16, 17], and suboptimal patient care [18]. Sleep regulation is highly susceptible to chronic stress exposure. Research has shown a relationship between sleep reduction, obesity, and increased body mass index [7, 19]. Chronic sleep disruption and circadian dysregulation, common in the practice of medicine, have been shown to be related to oxidative stress and cancers, heart disease, diabetes, aging, and neurodegenerative diseases. 
Physical activity and adequate sleep have been shown to mitigate the physiological effect of chronic stress. With competing demands, medical students have limited time for sleep and exercise. Medical schools should implement health promotion programs to help students to stay active and get sufficient sleep. However, knowledge of students' activity level and sleep habits and evidence-based interventions most likely to improve physical activity level and sleep quality and quantity among medical students are still lacking [10,11]. Recent technological advances in terms of sensors, and minimally invasive data capturing, represent a unique opportunity to determine and monitor physical activity and sleep. We hope to gain a better understanding of the need and the approach that would be required to establish a culture of health promotion and positive health behavior among our students.

The aim of the study was to (1) assess students' baseline activity level, sleep hours, and quantity in a cohort of first-year medical students and (2) examine whether the ability to track daily exercise and sleep hours with or without an incentive in the form of a game through a social media platform promoted students to achieve the recommended 10,000 steps per day and 7.5 to 9 daily sleep hours.

\section{Methods}

Participants Three hundred first-year medical students participated in the study (female $=173$, male $=127$, age 18 to 19 year old).

Institution The Yong Loo Lin School of Medicine, National University of Singapore, Singapore, is an undergraduate MBBS course. Medical training lasts 5 years, including a 2-year preclinical and a 3-year clinical training. The study was approved by the National University of Singapore Institutional Review Board with the number of NUS B-14-001.

Period of Study The study period was the second semester of academic year 2013/2014 (6 January 2014 to 20 April 2014).

Experimental Design It was a randomized controlled trial. Randomization was done by a computer program. Based on power calculation, a sample size of 86 per group is sufficient to detect a standard difference 0.5 , by using a 2 -sided 2 -sample $t$ test with $80 \%$ power and $1.66 \%$ significant level. Hence, our sample size of 100 per group was deemed sufficient to detect such a difference taking into consideration of the withdrawals. See Fig. 1 for details of the design. The control group received the educational modules with the rest of the participants on week 1, and started Fitbit Flex and the game on week 8. Experimental group 1 received the educational modules and Fitbit Flex on week 1, and started the game on week 8 .
Experimental group 2 received the educational modules, Fitbit Flex, and the game from week 1. The educational modules included importance of sleep in maintenance of longterm health, impacts of sleep disturbances on the brain and body, and physiological and psychological consequences of chronic stress on health. FitBit Flex records daily steps and distance as well as sleep. This game includes a log-in site for students and tutors to select their daily challenges, a leaderboard to track individual and houses' progress, and a forum for discussions and references on physical activity and sleep. Please refer to Fig. 2 for a snapshot of the game.

\section{Surveys}

International Physical Activity Questionnaire-Short Form $(I P A Q-S F)$ [20]: The IPAQ-SF includes seven questions on the types of activities and time they spent on physical activity in the past 7 days. The results were categorized and analyzed as low, moderate, and high physical activity.

Pittsburgh Sleep Quality Index (PSQI) [21]: Nineteen items generate seven component scores: subjective sleep quality, sleep latency, sleep duration, habitual sleep efficiency, sleep disturbances, use of sleep medication, and daytime dysfunction. The sum of scores yields one global score. The score $\leq 5$ indicates good sleep quality; a score $>5$ indicates poor quality.

The Perceived Stress Scale (PSS) [22]: The PSS measures the degree to which situations are appraised as stressful. The PSS showed adequate reliability, and the results were categorized as low, moderate, and high stress levels.

Tracking Device FitBit Flex is a wireless wearable sensor, recording daily walking steps, total distance, as well as sleep hours. The control group did not receive the device until week 8 , and the two experimental groups had the device during the course of study.

The Game Platform This platform included a log-in page for students to select their daily challenges in steps and sleep hours, a leader-board to track individual daily progress, and a forum for discussions and references on physical activity and sleep. During the first 8 weeks, the game platform was only available to the experimental group 2 . From week 9, all students had access to the game platform.

Data Analysis Descriptive analysis for data obtained from surveys and Fitbit Flex were conducted, including mean with $\mathrm{SD}$, or median with interquartile range for numerical variables, and number of participants and frequencies for categorical variables. Mixed model was applied to analyze students' daily steps and sleep time. The two-way interaction between groups and other factors such as weekday/weekend or gender 


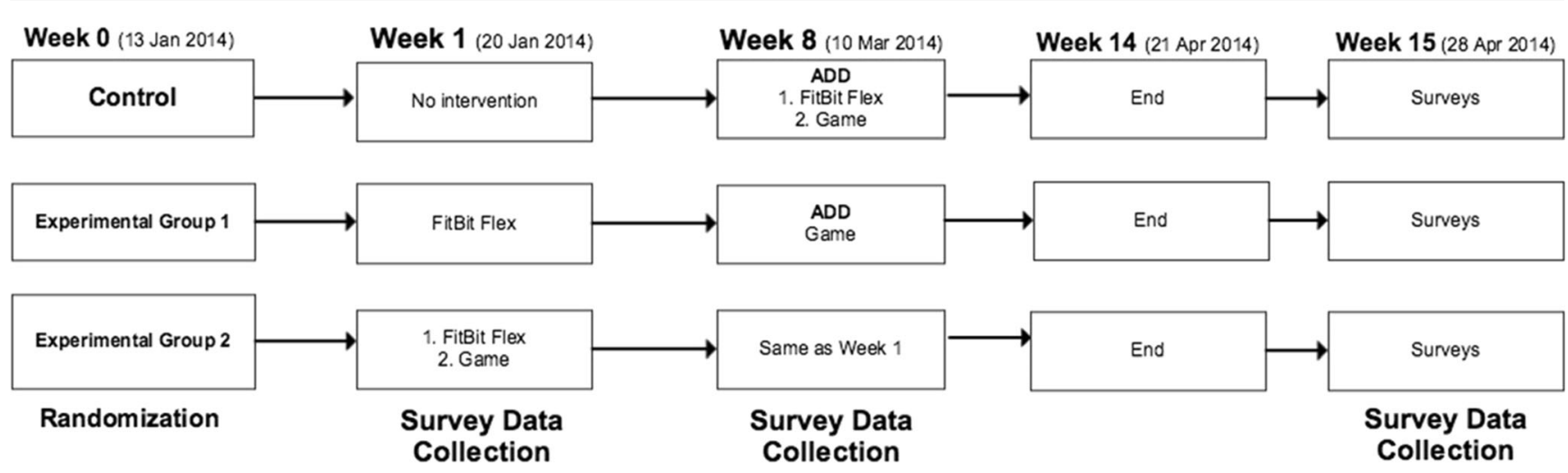

Fig. 1 The randomized controlled design of the study

were evaluated. If there was no significant interaction, only the main effects of the intervention group, time, and weekday/ weekend were included into the model. In case of significant interaction, subgroup analysis was conducted. Natural logarithm transformation was applied to daily steps. Chi-square test was done to compare students' sleep quality and exercise level based on the survey data. For data on sleep, sleep time $<2 \mathrm{~h}$ and $>16 \mathrm{~h}$ were removed from the data analysis. For data on daily steps, steps $<500$ and $>50,000$ were removed from the data analysis.

\section{Results}

\section{Physical Activity}

Survey Data Baseline survey showed that $85.0 \%$ of the students had moderate $(50.7 \%)$ and high $(34.3 \%)$ levels of activity. There was no statistical significant difference in physical activity levels among the three groups at week 1 , week 8 , and week 15 ( $p=0.473, p=0.893$, and $p=0.077$, respectively). Moreover, there was no significant increase in reported physical

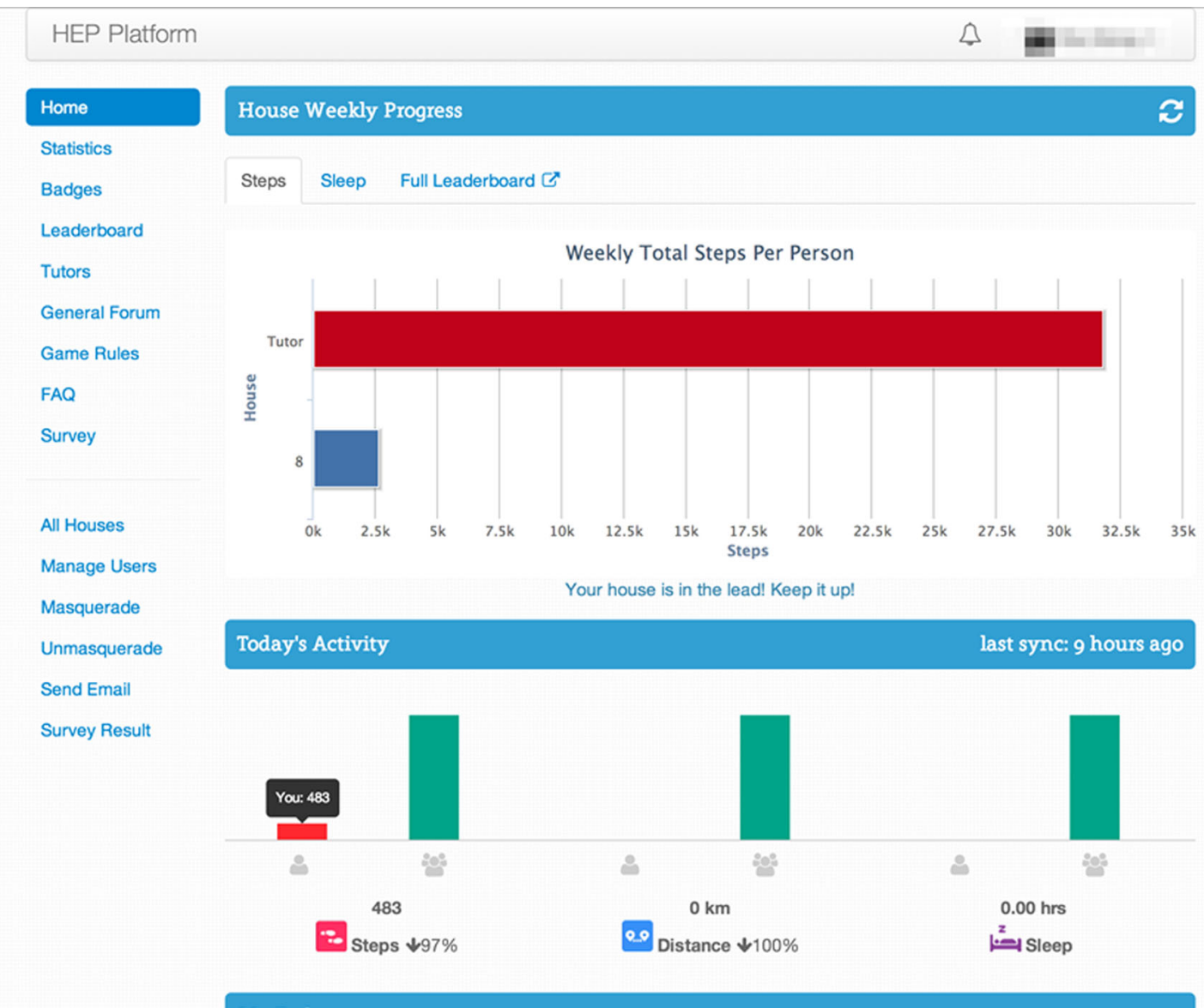

My Badges

Fig. 2 A snapshot of the gaming platform 
activity within each group at week 1,8 , and $15(p=0.469$, $p=0.222$, and $p=0.289$, respectively).

Fitbit Flex Data There was no significant difference in the baseline daily step counts between students in group 1 and those in group $2(p=0.823)$. Mixed model showed that students in group 2 (Fitbit and game) walked significantly more than those in group 1 (Fitbit alone) by $17.3 \%(p<0.0001)$. Daily step counts in week 9 were compared among the three groups, and the difference was statistically significant $(p=0.001)$. Students in group 1 walked significantly less compared with those in the control group by $15.9 \%$ ( $p=0.003$ ); however, there was no significant difference between group 2 and the control group $(p=0.456)$. Similarly, there was a statistically significant difference of daily step counts among the three groups from week 10 to week 14 $(p=0.006)$. Students in group 1 walked significantly less than those in the control group by $7.6 \%(p=0.0104)$, while there was no significant difference between group 2 and the control group ( $p=0.427$ ) (Table 1).

Students walked significantly more on weekdays compared with weekends in the three periods $(p<0.0001)$ by $27.5,40.0$, and $31.7 \%$, respectively. When weekdays and weekend step counts were analyzed separately from week 1 to week 8 , group 1 and group 2 had a statistically significant drop in daily step counts in week 6 and 7 (the recess and exam week) compared to weeks 1 to 5 . Group 1 showed a $25 \%$ decrease in daily step counts while group 2 decreased by $16 \%$.

\section{Sleep}

Sleep Time from the Survey Students reported daily sleep time from $400.8 \pm 63.8 \mathrm{~min}(6.68 \mathrm{~h})$ to $436.1 \pm 68.1 \mathrm{~min}$ (7.26 h). No statistically significant differences were found among the three groups at week 1, week 8, and week 15, and no significant differences were found in each group at week 1 , week 8 , and week 15 between weekdays and weekends.

Sleep Quality from the Survey Although no statistically significant differences among the three groups were found for week 1 and week 8 ( $p=0.569$ and $p=0.170$, respectively), week 15 data showed a significant difference in sleep quality among the three groups $(p=0.008)$, with $81.11 \%$ in group 1 and $85.88 \%$ in group 2 reporting a good quality of sleep, compared to $67.05 \%$ in the control group.

FitBit Data The mean daily sleep time was summarized in Table 2. There was no significant difference in sleep time at baseline between group 1 and group $2(p=0.204)$. Mixed model showed that there was no significant difference of daily sleep time from week 2 to week 8 between group 1 and group $2(p=0.788)$. The daily sleep time at week 9 was compared among the three groups, and the result showed that there was no statistical difference either $(p=0.130)$. However, we observed that students in group 1 (Fitbit alone) slept 21.7 min longer than those in the control group $(p=0.073)$; students in group 2 (Fitbit plus game) slept 18.4 min longer than the control group $(p=0.148)$. During week 10 to week 14 , there was significant difference of daily sleep time among the three groups $(p=0.034)$. Students in group 1 slept significantly longer than those in the control group $(p=0.017)$ by 17.5 min. Students in group 2 slept 14.4 min longer than those in the control group; however, it was just boundary significant $(p=0.064)$. It was observed that students always slept significantly longer during weekends compared to weekdays in the three periods $(p<0.0001)$ by $63.4,59.2$, and $51.2 \mathrm{~min}$, respectively. By week 14, all students' (average of all groups) sleep time reached on average $7.90 \mathrm{~h}$ during weekdays and $8.04 \mathrm{~h}$ during weekends.

Subgroup analysis showed that being able to track one's daily sleep hours increased significantly sleep time in all three groups ( $p<0.000$ for all three groups). Compared to week 1 , group 1 (FitBit alone) and group 2 (FitBit + game) slept significantly longer from week 5 onwards; by week 14, group 1 slept $69.85 \mathrm{~min}$ longer and group 2 slept $28.9 \mathrm{~min}$ longer $(p<0.000)$.

From week 9 to week 14, all students including the control group had access to FitBit Flex and the game; no significant differences in sleep time were found between group 1 and group $2(p=0.655)$. At week 9, group 1 average sleep time during the weekday was $449.23 \mathrm{~min}(7.48 \mathrm{~h}) \pm \mathrm{SD} 60.70$, weekend $486.05 \mathrm{~min}$ $(8.10 \mathrm{~h}) \pm$ SD 87.64 while group 2 average sleep time was $429.42 \mathrm{~min}(7.15 \mathrm{~h}) \pm$ SD 91.44 for weekdays and $471.00 \mathrm{~min}(7.81 \mathrm{~h}) \pm 90.35$ for weekends. By week 14, group 1 increased sleep time by 15 min during weekdays, 17 min during weekends and group 2 by $13 \mathrm{~min}$ during the weekend and 49 min during weekdays. From weeks 9 to 14 , group 1 slept 17.4 min more $(p=0.015)$ and group $213.8 \mathrm{~min}(p=0.070)$ more than the control group. For the control group, between week 9 and week 14 during weekdays, sleep time increased significantly from week 12 onwards with an overall 61-min increase. Stress

Survey Data There was no statistical significant difference in stress scores among the three groups in the three data collection points $(p=0.073, p=0.259$, and $p=0.357$, respectively). The survey score lower than 13 is deemed as low stress, 14-19 as medium, and above 20 as high. Table 3 summarizes the results. 
Table 1 Daily steps recorded by FitBit Flex

\begin{tabular}{|c|c|c|c|c|c|c|}
\hline & \multicolumn{2}{|c|}{ Control group } & \multicolumn{2}{|c|}{ Group 1 (FitBit alone) } & \multicolumn{2}{|c|}{ Group 2 (FitBit + game) } \\
\hline & Weekday & Weekend & Weekday & Weekend & Weekday & Weekend \\
\hline Week 1 & & & $11,424(5971)$ & $8171(5051)$ & 9979(5077) & $8666(5736)$ \\
\hline Week 2 & & & $9444(3826)$ & $8225(5604)$ & $10,176(5773)$ & $8685(6476)$ \\
\hline Week 3 & & & $8208(4642)$ & $5316(4357)$ & $9406(5095)$ & $6496(6089)$ \\
\hline Week 4 & & & $8154(4637)$ & $5967(6161)$ & $9025(5743)$ & $8252(4787)$ \\
\hline Week 5 & & & $9008(3932)$ & $6221(3941)$ & $9645(5979)$ & $7531(5242)$ \\
\hline Week 6 & & & $6386(3427)$ & $5126(5480)$ & $8270(4719)$ & $6455(4940)$ \\
\hline \multicolumn{7}{|l|}{ (recess) } \\
\hline \multicolumn{7}{|l|}{ (exam week) } \\
\hline Week 8 & & & $8428(3876)$ & $7591(5070)$ & $9470(7243)$ & $7585(6078)$ \\
\hline \multirow[t]{2}{*}{ Week 9} & 9801 & 8050 & $8574(3131)$ & $5689(5456)$ & $8817(4772)$ & $7353(6662)$ \\
\hline & $(4285)$ & $(6029)$ & & & & \\
\hline \multirow[t]{2}{*}{ Week 10} & $9805(4286)$ & 7976 & $8490(3198)$ & $6934(4785)$ & $8818(5156)$ & $9714(4959)$ \\
\hline & & (5574) & & & & \\
\hline \multirow[t]{2}{*}{ Week 11} & 9280 & 7499 & $8499(2881)$ & $6129(6020)$ & $9387(4242)$ & $9291(4693)$ \\
\hline & $(3748)$ & $(6518)$ & & & & \\
\hline \multirow[t]{2}{*}{ Week 12} & 8518 & 6893 & $7878(3568)$ & $6498(5507)$ & $8261(5125)$ & $5852(5059)$ \\
\hline & $(3030)$ & $(6193)$ & & & & \\
\hline Week 13 & 7593 & 6368 & 7955(3070) & $6019(6914)$ & $8142(4776)$ & $5920(7568)$ \\
\hline (study week) & (4371) & (4903) & & & & \\
\hline Week 14 & 7719 & 5595 & $6051(2423)$ & $5696(6878)$ & 7585(3329) & $6163(5902)$ \\
\hline (study week) & (4086) & (4927) & & & & \\
\hline
\end{tabular}

Values are median daily steps (interquartile range), weekday (Monday to Friday), and weekend (Saturday and Sunday)

\section{Discussion}

Physical Activity The recommended daily walking for adults is 10,000 steps, and our students walked close to 10,000 steps daily. Group comparison of steps from week 1 to week 8 showed that students who had access to both Fitbit Flex and game walked an average of 728 steps more than those who only had access to Fitbit Flex. However, this effect was not sustained from week 9 to week 14. Overall, self-reported data and data from the tracking device showed that students were active, and the interventions did not significantly increase their activity level except during recess and exam time. Instead of motivating students to start doing exercise, the interventions should reinforce the benefit of maintaining a good level of daily exercise especially during exam and recess weeks.

Sleep Quality and Quantity According to the US National Sleep Foundation's recommendation, the daily sleep hours for 18-20 years old is 7.5 to $9 \mathrm{~h}$ [23]. At baseline, our students slept an average of $6.5 \mathrm{~h}$. Interestingly, being able to track sleep time influenced students' behavior. By the end of the study, all students had significantly increased their sleep time to 7.5 to $8.0 \mathrm{~h}$, and the two experimental groups reported a better sleep quality. Overall, monitoring sleep pattern helped students to achieve and maintain the recommended daily sleep hours of 7.5 to $9 \mathrm{~h}$.

Stress More than half of students' stress levels were medium to high from week 1, and the similar pattern persisted until week 14. Medical students' stress is affected by multiple factors, for example, academic and clinical pressures. Minor changes in students' activity level and sleep hours were not sufficient to reduce their stress level. On the other hand, stress was measured through the self-report survey. Physiological measurements of stress could more accurately demonstrate objective stress responses to changes in students' daily activity and sleep time.

Tools to Monitor Students' Physical Activity and Sleep This study demonstrated the possibility and benefits of objective measurements of physical activity and sleep, as well as implications for health promotion interventions. Access to tracking devices has the potential to promote students to maintain a good level of exercise and regulate daily sleep. Sensor technology and large data sets generated by the technology should be fully utilized in students' health behavior promotion. 
Table 2 Weekly mean sleep time from FitBit Flex

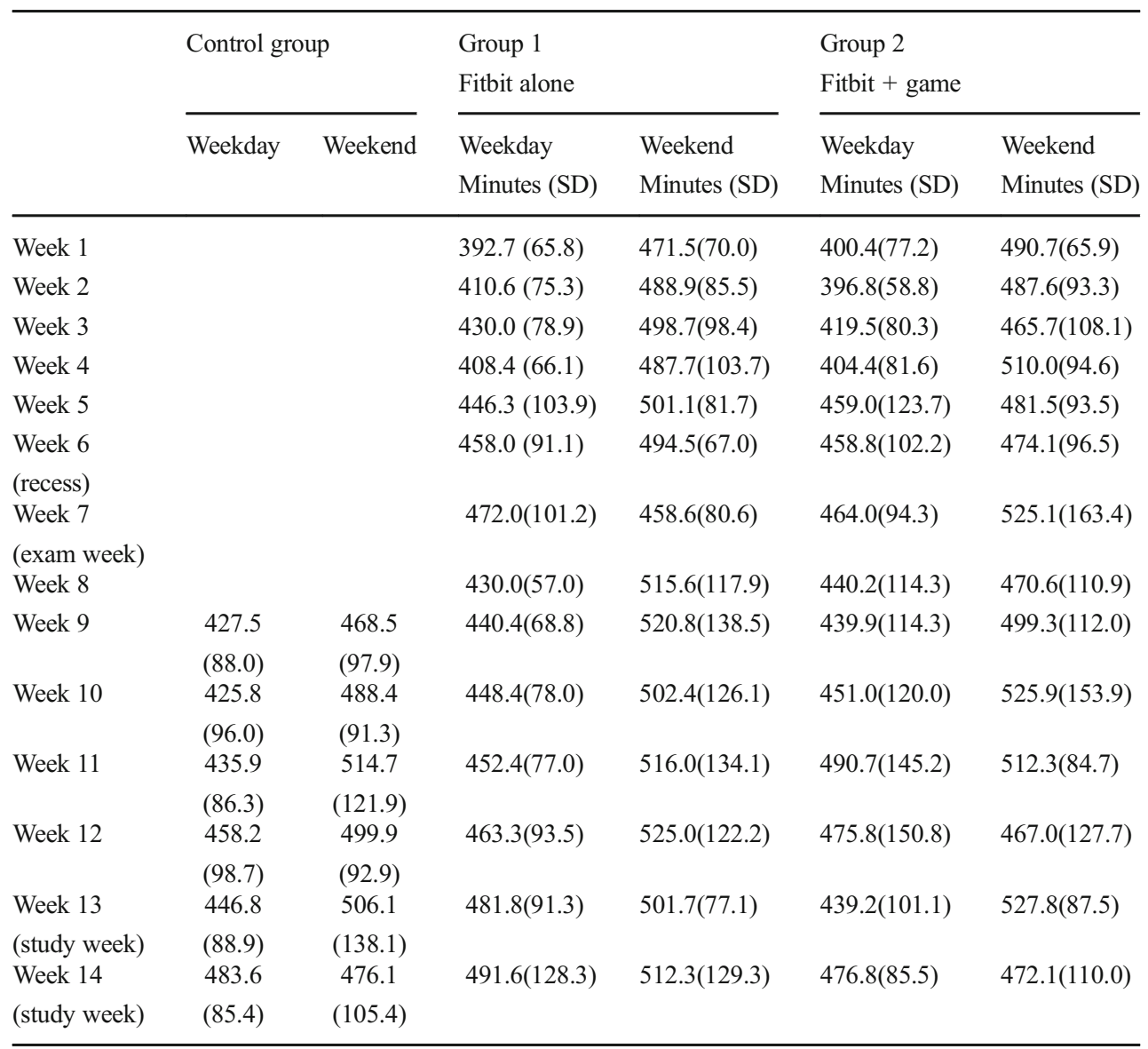

Values are mean in minutes (SD), weekday (Monday to Friday), and weekend (Saturday and Sunday)
It is beneficial for medical students to understand how these devices work and what the data means, and to experience how difficult it could be in maintaining healthy behaviors. Maintaining healthy behavior is essential for performance, professionalism, and patient safety.

Study Limitations The lack of a blind device for the control group (a sensor measuring activity and sleep without allowing data access) proved impossible in the given context. Hence, no objective data was available for the control group from week 1 to week 8 .

Future Directions Further research should explore participants' individual responses to interventions and to identify the most influencing factor(s) in regards to adherence to healthy behaviors. This will inform schools on contextual factors such as location, type of classroom settings, and building designs to promote healthy behaviors. Long-term adherence

Table 3 Students perceived stress level based on the Perceived Stress Scale

\begin{tabular}{|c|c|c|c|c|c|c|c|c|c|}
\hline & \multicolumn{3}{|l|}{ Week 1} & \multicolumn{3}{|l|}{ Week 8} & \multicolumn{3}{|l|}{ Week 14} \\
\hline & $\begin{array}{l}\leq 13 \\
\text { Frequency } \\
(\%)\end{array}$ & $\begin{array}{l}14-19 \\
\text { Frequency } \\
(\%)\end{array}$ & $\begin{array}{l}\geq 20 \\
\text { Frequency } \\
(\%)\end{array}$ & $\begin{array}{l}\leq 13 \\
\text { Frequency } \\
(\%)\end{array}$ & $\begin{array}{l}14-19 \\
\text { Frequency } \\
(\%)\end{array}$ & $\begin{array}{l}\geq 20 \\
\text { Frequency } \\
(\%)\end{array}$ & $\begin{array}{l}\leq 13 \\
\text { Frequency } \\
(\%)\end{array}$ & $\begin{array}{l}14-19 \\
\text { Frequency } \\
(\%)\end{array}$ & $\begin{array}{l}\geq 20 \\
\text { Frequency } \\
(\%)\end{array}$ \\
\hline $\begin{array}{l}\text { Control } \\
\text { group }\end{array}$ & $\begin{array}{l}32 \\
(35.2)\end{array}$ & $\begin{array}{l}36 \\
(39.6)\end{array}$ & $\begin{array}{l}23 \\
(25.3)\end{array}$ & $\begin{array}{l}36 \\
(38.7)\end{array}$ & $\begin{array}{l}25 \\
(26.9)\end{array}$ & $\begin{array}{l}32 \\
(34.4)\end{array}$ & $\begin{array}{l}37 \\
(41.1)\end{array}$ & $\begin{array}{l}23 \\
(25.6)\end{array}$ & $\begin{array}{l}30 \\
(34.4)\end{array}$ \\
\hline Group 1 & $\begin{array}{l}49 \\
(44.1)\end{array}$ & $\begin{array}{l}31 \\
(42.6)\end{array}$ & $\begin{array}{l}15 \\
(15.8)\end{array}$ & $\begin{array}{l}41 \\
(44.1)\end{array}$ & $\begin{array}{l}23 \\
(24.7)\end{array}$ & $\begin{array}{l}29 \\
(31.2)\end{array}$ & $\begin{array}{l}37 \\
(42.0)\end{array}$ & $\begin{array}{l}30 \\
(34.1)\end{array}$ & $\begin{array}{l}21 \\
(23.9)\end{array}$ \\
\hline Group 2 & $\begin{array}{l}35 \\
(39.8)\end{array}$ & $\begin{array}{l}33 \\
(37.5)\end{array}$ & $\begin{array}{l}20 \\
(22.7)\end{array}$ & $\begin{array}{l}30 \\
(33.7)\end{array}$ & $\begin{array}{l}30 \\
(33.7)\end{array}$ & $\begin{array}{l}29 \\
(32.6)\end{array}$ & $\begin{array}{l}38 \\
(41.3)\end{array}$ & $\begin{array}{l}34 \\
(37.0)\end{array}$ & $\begin{array}{l}20 \\
(21.7)\end{array}$ \\
\hline
\end{tabular}

Values are $n(\%) ; \leq 13$ refers to low stress, $14-19$ for medium stress, and $\geq 20$ for high stress 
to positive health routines depends on a positive health culture in educational environments.

Acknowledgments The authors would like to acknowledge the administrative support of the Student Affair team Yong Loo Ling School of Medicine, National University of Singapore, Singapore.

Author's Contribution MVC contributed to the conception, the design, the implementation of the study, and the writing of the final version of the manuscript. FHG contributed to the conception, the design, the implementation of the study and the writing of the IRB, and the first draft of the manuscript. MB gave some input on the data analysis. BL designed the game and was responsible for the acquisition of the FitBit data. LS did all the statistical analysis. CD contributed to the writing of the IRB and the writing and submission of the final version of the paper. All authors approved the final manuscript for publication.

\section{Compliance with Ethical Standards}

Funding This study was funded by the Dean's office of the Yong Loo Lin medical school, Student Affairs team budget.

Ethical Approval The National University of Singapore Institutional Review Board approved this study.

Open Access This article is distributed under the terms of the Creative Commons Attribution 4.0 International License (http:// creativecommons.org/licenses/by/4.0/), which permits unrestricted use, distribution, and reproduction in any medium, provided you give appropriate credit to the original author(s) and the source, provide a link to the Creative Commons license, and indicate if changes were made.

\section{References}

1. Singh G, Hankins M, Weinman JA. Does medical school cause health anxiety and worry in medical students? Med Educ. 2004;38(5):479-81.

2. Guthrie EA, Black D, Shaw CM, Hamilton J, Creed FH, Tomenson B. Embarking upon a medical career: psychological morbidity in first year medical students. Med Educ. 1995;29(5):337-41.

3. Ishak W, Nikravesh R, Lederer S, Perry R, Ogunyemi D, Bernstein C. Burnout in medical students: a systematic review. Clin Teach. 2013;10(4):242-5.

4. Dyrbye LN, West CP, Satele D, Boone S, Tan L, Sloan J, et al. Burnout among U.S. medical students, residents, and early career physicians relative to the general U.S. population. Acad Med. 2014;89(3):443-51.

5. Hassed C, De Lisle S, Sullivan G, Pier C. Enhancing the health of medical students: outcomes of an integrated mindfulness and lifestyle program. Adv Heal Sci Educ. 2009;14(3):387-98.

6. McEwen BS. Central effects of stress hormones in health and disease: Understanding the protective and damaging effects of stress and stress mediators. European Journal of Pharmacology. 2008. p. 174-85.

7. McEwen BS. Protective and damaging effects of stress mediators: central role of the brain. Dialogues Clin Neurosci. 2006;8(4):367-81.

8. Pinto P, Geoffroy M, Power C. Depressive symptoms and physical activity during 3 decades in adult life: bidirectional associations in a prospective cohort study. JAMA Psychiatry. 2014;71(12):1373-80.

9. Firth-Cozens J, Greenhalgh J. Doctors' perceptions of the links between stress and lowered clinical care. Soc Sci Med. 1997;44(7):1017-22.

10. Sexton JB, Thomas EJ, Helmreich RL. Error, stress, and teamwork in medicine and aviation: cross sectional surveys. BMJ. 2000;320(7237):745-9.

11. West CP, Dyrbye LN, Rabatin JT, Call TG, Davidson JH, Multari $\mathrm{A}$, et al. Intervention to promote physician well-being, job satisfaction, and professionalism: a randomized clinical trial. JAMA Intern Med. 2014;174(4):527-33.

12. Chen KY, Yang CM, Lien CH, Chiou HY, Lin MR, Chang HR, et al. Burnout, job satisfaction, and medical malpractice among physicians. Int J Med Sci. 2013;10(11):1471-8.

13. Jones JW, Barge BN, Steffy BD, Fay LM, Kunz LK, Wuebker LJ. Stress and medical malpractice: organizational risk assessment and intervention. J Appl Psychol. 1988;73(4):727-35.

14. DiMatteo MR, Sherbourne CD, Hays RD, Ordway L, Kravitz RL, McGlynn EA, et al. Physicians' characteristics influence patients' adherence to medical treatment: results from the Medical Outcomes Study. Health Psychol. 1993;12(2):93-102.

15. Halbesleben JR, Rathert C. Linking physician burnout and patient outcomes: exploring the dyadic relationship between physicians and patients. Health Care Manag Rev. 2008;33(1):29-39.

16. Dyrbye LN, Shanafelt TD. Physician burnout: a potential threat to successful health care reform. J Am Med Assoc. 2011;305(19): 2009-10.

17. Weng HC, Hung CM, Liu YT, Cheng YJ, Yen CY, Chang CC, et al. Associations between emotional intelligence and doctor burnout, job satisfaction and patient satisfaction. Med Educ. 2011;45(8):835-42.

18. El-Bar N, Levy A, Wald HS, Biderman A. Compassion fatigue, burnout and compassion satisfaction among family physicians in the Negev area - a cross-sectional study. Isr J Health Policy Res. Israel Journal of Health Policy Research; 2013; 2(1):31.

19. McEwen BS. Central effects of stress hormones in health and disease: understanding the protective and damaging effects of stress and stress mediators. Eur J Pharmacol. 2008;583(2-3):174-85.

20. Craig CL, Marshall AL, Sjöström M, Bauman AE, Booth ML, Ainsworth BE, et al. International physical activity questionnaire: 12 -country reliability and validity. Med Sci Sports Exerc. 2003;35(8):1381-95.

21. Buysse DJ, Reynolds CF, Monk TH, Berman SR, Kupfer DJ. The Pittsburgh Sleep Quality Index: a new instrument for psychiatric practice and research. Psychiatry Res. 1989;28(2):193-213.

22. Cohen S, Kamarck T, Mermelstein R. A global measure of perceived stress. J Health Soc Behav. 1983;24(4):385-96.

23. Hirshkowitz M, Whiton K, Albert SM, Alessi C, Bruni O, DonCarlos L, et al. National Sleep Foundation's sleep time duration recommendations: methodology and results summary. Sleep Heal. 2015;1(1):40-3. 